\title{
Article \\ Quinacrine Ameliorates Cisplatin-Induced Renal Toxicity via Modulation of Sirtuin-1 Pathway
}

\author{
Nada F. Abo El-Magd ${ }^{1, *(\mathbb{C})}$, Hasnaa Ali Ebrahim ${ }^{2,3}$, Mohamed El-Sherbiny ${ }^{4} \mathbb{C}^{\text {and Nada H. Eisa }}{ }^{1, *}$ \\ 1 Biochemistry Department, Faculty of Pharmacy, Mansoura University, Mansoura 35516, Egypt \\ 2 Department of Basic Medical Sciences, College of Medicine, Princess Nourah Bint Abdulrahman University, \\ Riyadh 11564, Saudi Arabia; haebrahim@pnu.edu.sa \\ 3 Department of Anatomy, Faculty of Medicine, Mansoura University, Mansoura 35516, Egypt \\ 4 Department of Basic Medical Sciences, College of Medicine, AlMaarefa University, \\ Riyadh 13713, Saudi Arabia; msharbini@mcst.edu.sa \\ * Correspondence: dr.nada.fawzy@gmail.com (N.F.A.E.-M.); nadaeisa@hotmail.com (N.H.E.)
}

Citation: Abo El-Magd, N.F.

Ebrahim, H.A.; El-Sherbiny, M.; Eisa, N.H. Quinacrine Ameliorates Cisplatin-Induced Renal Toxicity via Modulation of Sirtuin-1 Pathway. Int. J. Mol. Sci. 2021, 22, 10660. https:// doi.org/10.3390/ijms221910660

Academic Editor: Hajime Nagasu

Received: 27 August 2021

Accepted: 28 September 2021

Published: 1 October 2021

Publisher's Note: MDPI stays neutral with regard to jurisdictional claims in published maps and institutional affiliations.

Copyright: (c) 2021 by the authors. Licensee MDPI, Basel, Switzerland. This article is an open access article distributed under the terms and conditions of the Creative Commons Attribution (CC BY) license (https:/ / creativecommons.org/licenses/by/ $4.0 /)$.

\begin{abstract}
Renal toxicity is a serious side effect that hinders the use of cisplatin, a commonly used and effective chemotherapeutic agent. Meanwhile, quinacrine is an FDA approved drug that has been stated for its anti-inflammatory effect. Thus, we investigated the ameliorative effect of quinacrine against cisplatin-induced renal toxicity. Single intraperitoneal (i.p.) $10 \mathrm{mg} / \mathrm{kg}$ cisplatin administration induced renal injury in rats. Our results showed that $10 \mathrm{mg} / \mathrm{kg} /$ day quinacrine decreased the mortality rate of rats from $46.15 \%$ (cisplatin group) to $12.5 \%$, and significantly decreased renal tissue fibrosis, relative kidney to body weight ratio, serum creatinine and urea levels compared with the cisplatin group. Indeed, quinacrine significantly decreased renal malondialdehyde concentration and increased renal total antioxidant capacity, compared with the cisplatin group. Furthermore, quinacrine caused significant upregulation of renal sirtuin-1 (SIRT-1) with significant downregulation of intercellular adhesion molecule-1 (ICAM-1) and tumor necrosis factor- $\alpha$ (TNF- $\alpha$ ). Moreover, quinacrine significantly blocked cisplatin-induced apoptosis, which was made evident by downregulating renal apoptotic proteins (BAX and p53) and upregulating the renal anti-apoptotic protein BCL2, compared with the cisplatin group. In conclusion, this study demonstrates, for the first time, that quinacrine alleviates cisplatin-induced renal toxicity via upregulating SIRT-1, downregulating inflammatory markers (ICAM-1 and TNF- $\alpha$ ), reducing oxidative stress, and inhibiting apoptosis.
\end{abstract}

Keywords: quinacrine; SIRT-1; nephrotoxicity; inflammation; apoptosis

\section{Introduction}

Cisplatin is an efficient chemotherapeutic agent in in the treatment of different types of cancers, including non-small cell lung carcinoma, cervical, ovarian, testicular and head and neck cancers [1-3]. Unfortunately, cisplatin clinical use is accompanied with serious side effects on various normal tissues [4]. Cisplatin accumulates in renal proximal tubules, causing acute kidney injury [5]. Approximately 50\% of cancer patients undergoing cisplatin chemotherapy suffer from renal dysfunction [6]. Hence, finding an agent that alleviates cisplatin-induced renal injury is of great importance for cisplatin-treated cancer patients.

It has been shown that cisplatin induces renal injury via increasing oxidative stress levels, mitochondrial dysfunction, inflammation, DNA damage and apoptosis [7]. Sirtuin-1 (SIRT-1), a NAD ${ }^{+}$dependent histone deacetylase, has recently gained considerable attention, due to its differential regulation of inflammation, stress response and apoptosis [8,9]. SIRT1 has been shown to play a key role in cisplatin-induced apoptosis via deacetylation of p53 [10,11]. Thus, SIRT-1 represents an attractive therapeutic target for inflammatorymediated renal injuries $[9,12]$.

Quinacrine is an FDA-approved antimalarial drug and a well-known anti-inflammatory agent. It has also been reported to be beneficial in managing cancer, lupus erythemato- 
sus, and cutaneous sarcoidosis [13-15]. Mechanistically, several studies have reported the link between quinacrine and inflammation via p53 and superoxide dismutase activation as well as NF-KB and phospholipase A2 inhibition. However, no studies have investigated the role of quinacrine in alleviating cisplatin-indued renal damage via regulation of the SIRT-1 pathway. Consequently, in the current study, we hypothesize that quinacrine might alleviate cisplatin-induced acute renal injury via differential regulation of the SIRT-1/inflammatory/apoptotic axis.

\section{Results}

\subsection{Quinacrine Attenuated Cisplatin-Induced Mortality, Nephrotoxicity and Oxidative Stress}

Cisplatin induced mortality in $46.2 \%$ of rats (Figure $1 \mathrm{~A}$ ) along with inducing a significant increase in the relative kidney to body weight ratio (Figure 1B), serum creatinine (Figure 1C), and urea (Figure 1D) concentrations, compared to the normal group. Quinacrine protected rats from these nephrotoxic effects as indicated by decreasing the mortality rate to $12.5 \%$ (Figure 1A). Moreover, quinacrine caused a significant decrease in the relative kidney to rat body weight ratio (Figure 1B), serum creatinine (Figure 1C) and urea (Figure 1D) concentrations, compared with the cisplatin group.

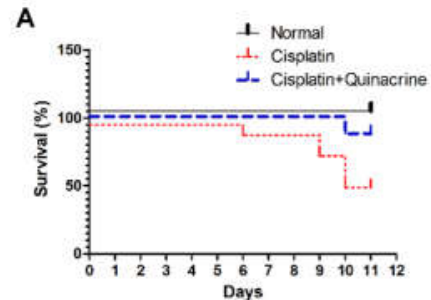

D

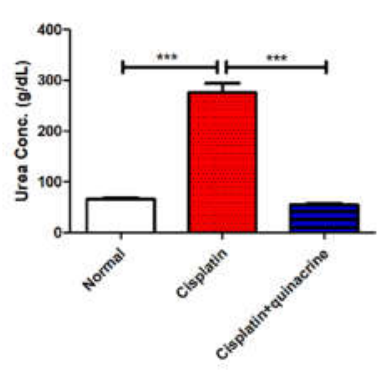

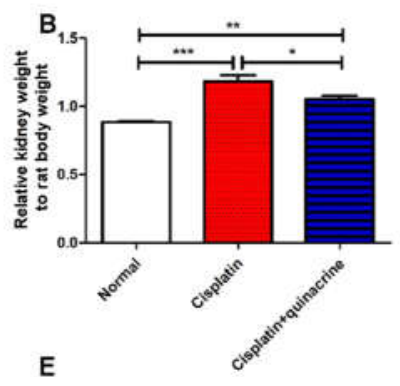

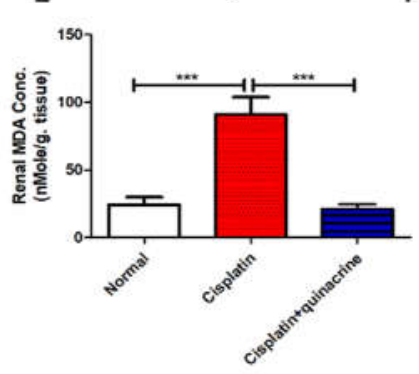

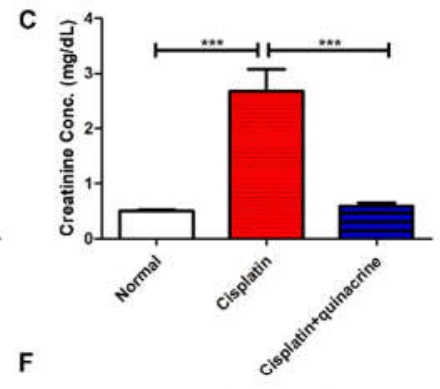

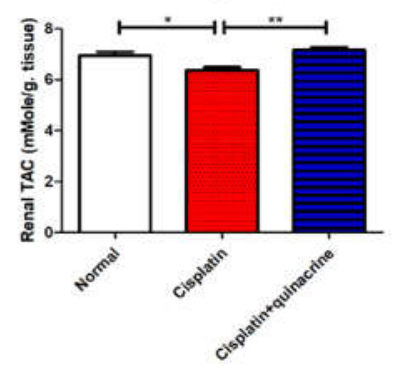

Figure 1. Quinacrine attenuated cisplatin-induced mortality, nephrotoxicity and oxidative stress. Effect of 10 days' administration of quinacrine $(10 \mathrm{mg} / \mathrm{kg} / \mathrm{rat} /$ day, intraperitoneal $)$ against cisplatininduced nephrotoxicity in rats on (A) \% survival $(n=7-8)$, (B) relative kidney to rat body weight ratio $(n=7-8),(\mathbf{C})$ serum creatinine concentration, $(\mathbf{D})$ serum urea concentration $(n=7-8)$, (E) renal malondialdehyde (MDA) concentration $(n=7-8)$ and $(\mathbf{F})$ renal total antioxidant capacity (TAC) $(n=7-8) .{ }^{*}: p<0.05 ;{ }^{* *}: p<0.01$ and ${ }^{* * *} p<0.001$. Data are expressed as mean \pm SEM.

Cisplatin induced oxidative stress in renal tissues, as it significantly increased renal malondialdehyde (MDA) concentration (Figure 1E) and significantly decreased total antioxidant capacity (TAC) (Figure 1F), compared to the normal group. Quinacrine protected against cisplatin-induced oxidative stress, as it significantly decreased renal MDA concentration (Figure 1E) and significantly increased TAC (Figure 1F), compared with the cisplatin group.

\subsection{Quinacrine Attenuated Cisplatin-Induced Renal Structure Alteration and Fibrosis}

Cisplatin induced tubular dilation lined with flattened epithelium and vacuolar degeneration in the renal cortex (Figure 2A), and marked tubular dilation, hydropic degeneration, and necrosis in renal medulla (Figure 2B). Moreover, cisplatin significantly increased the area of fibrosis (Figure 2C,D), compared to the normal group. Quinacrine attenuated 
cisplatin-induced fibrosis and structural changes. This was evident by mild tubular dilation lined with flattened epithelium in the renal cortex (Figure 2A), moderate tubular dilation in the renal medulla (Figure 2B), and significant decrease in the percentage of fibrotic area (Figure 2C,D), compared to the cisplatin group. Although the quinacrine treatment greatly improved renal structural organization compared to the cisplatin group, it could not completely reverse cisplatin-induced renal degeneration (Figure 2A,B).
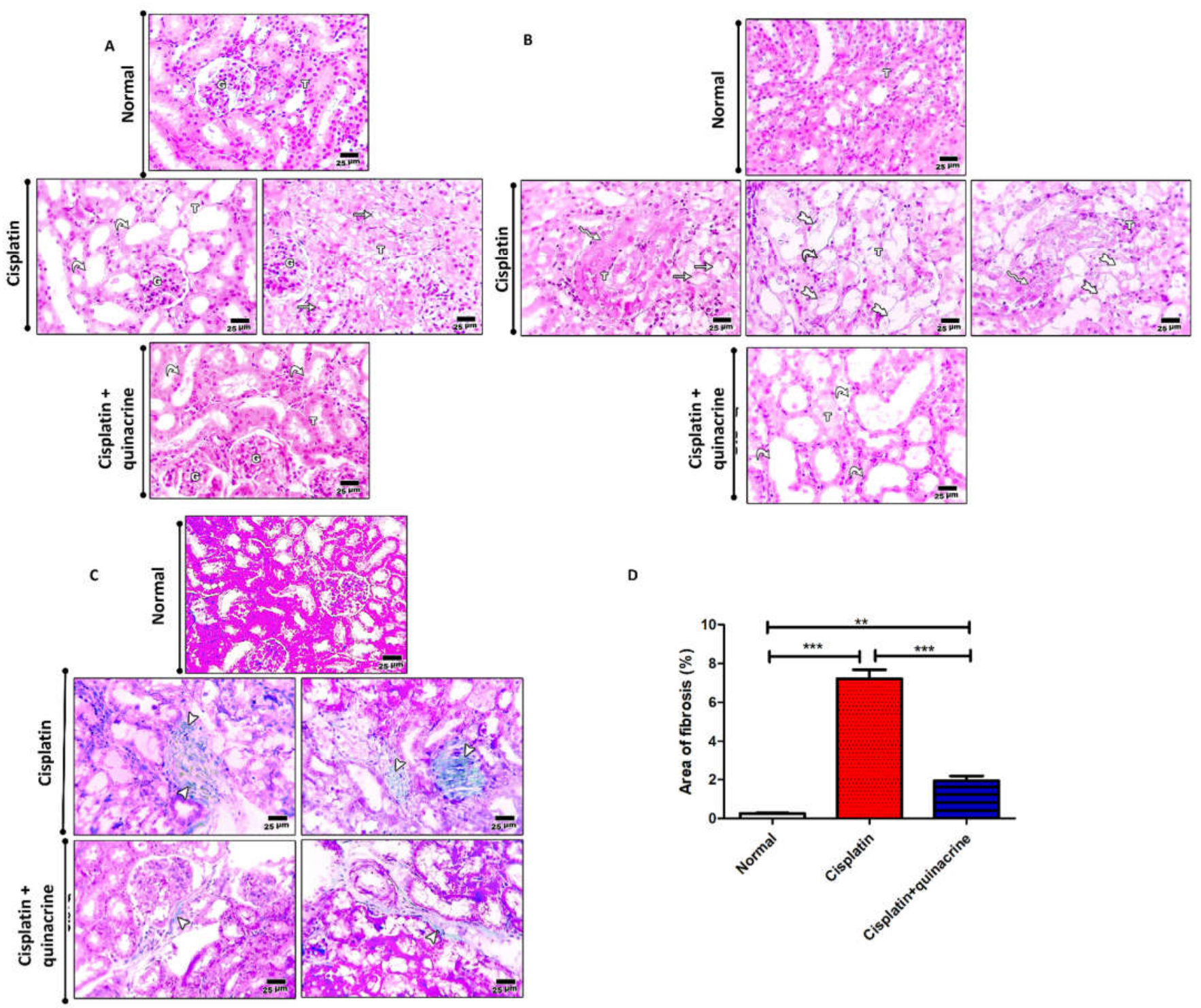

D

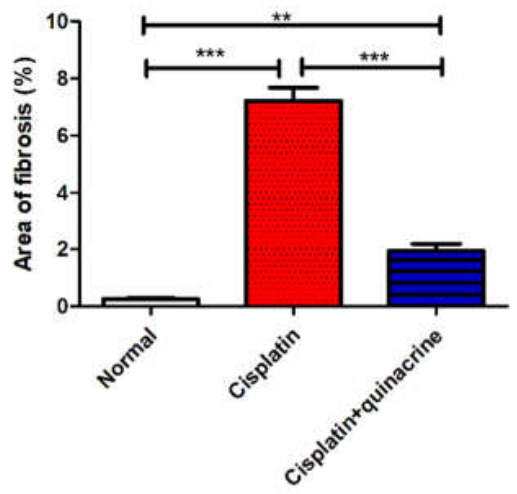

Figure 2. Quinacrine attenuated cisplatin-induced renal structure alteration and fibrosis. (A) Microscopic pictures of H\&E-stained renal sections showing normal cortex including glomeruli $(G)$ and tubules $(T)$ in normal group, tubular dilation lined with flattened epithelium (curved arrows), vacuolar degenerative (arrows) in cortex of cisplatin group and mild tubular dilation lined with cuboidal epithelium (curved arrows) in cortex of cisplatin + quinacrine treated group. (B) Microscopic pictures of H\&E-stained renal sections showing normal medulla cortex in normal group, marked tubular dilation (curved arrows), hydropic degeneration (rocket arrows) and necrosis (wavy arrows) in medulla in cisplatin group and moderate tubular dilation (curved arrows) in medulla of cisplatin + quinacrine group. (C) Microscopic pictures of Masson trichrome stained renal sections showing no fibrosis in normal group, bluish green fibrous tissue deposition (arrowheads) in renal sections from cisplatin group and the fibrous tissue deposition markedly decreased in cisplatin + quinacrine group. High magnification X: 400 bar 25. (D) The effect on area of fibrosis (\%) of quinacrine administration $\left(10 \mathrm{mg} / \mathrm{kg} / \mathrm{rat} /\right.$ day: IP) against cisplatin-induced nephrotoxicity in rats $(n=6){ }^{* *}: p<0.01$ and ${ }^{* * *} p<0.001$. Data are expressed as mean \pm SEM. 


\subsection{Quinacrine Attenuated Cisplatin-Induced Dysregulation of SIRT-1, ICAM-1 and TNF- $\alpha$}

Cisplatin significantly decreased renal SIRT-1 concentration (Figure 3A) and significantly increased renal intercellular adhesion molecule-1 (ICAM-1) concertation (Figure 3B), compared with the normal group. Moreover, cisplatin significantly increased renal tumor necrosis factor- $\alpha$ (TNF- $\alpha$ ) concentration (Figure 3C-E), compared to the normal group. Interestingly, quinacrine successfully attenuated cisplatin-induced dysregulation of the above-mentioned markers. Quinacrine significantly upregulated renal SIRT-1 concentration (Figure 3A), and significantly downregulated renal ICAM-1 (Figure 3B) and TNF- $\alpha$ (Figure $3 \mathrm{C}-\mathrm{E}$ ) concentrations, compared to the cisplatin group.

A

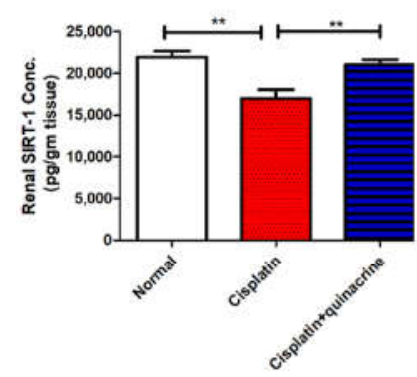

C

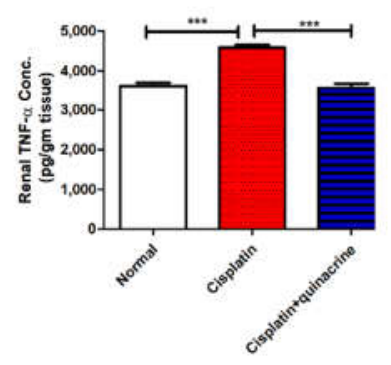

B

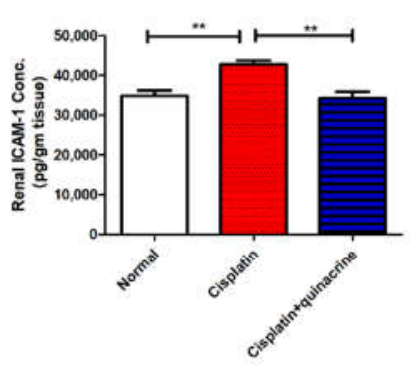

D

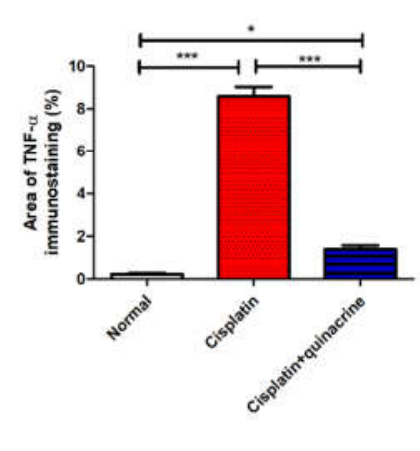

E

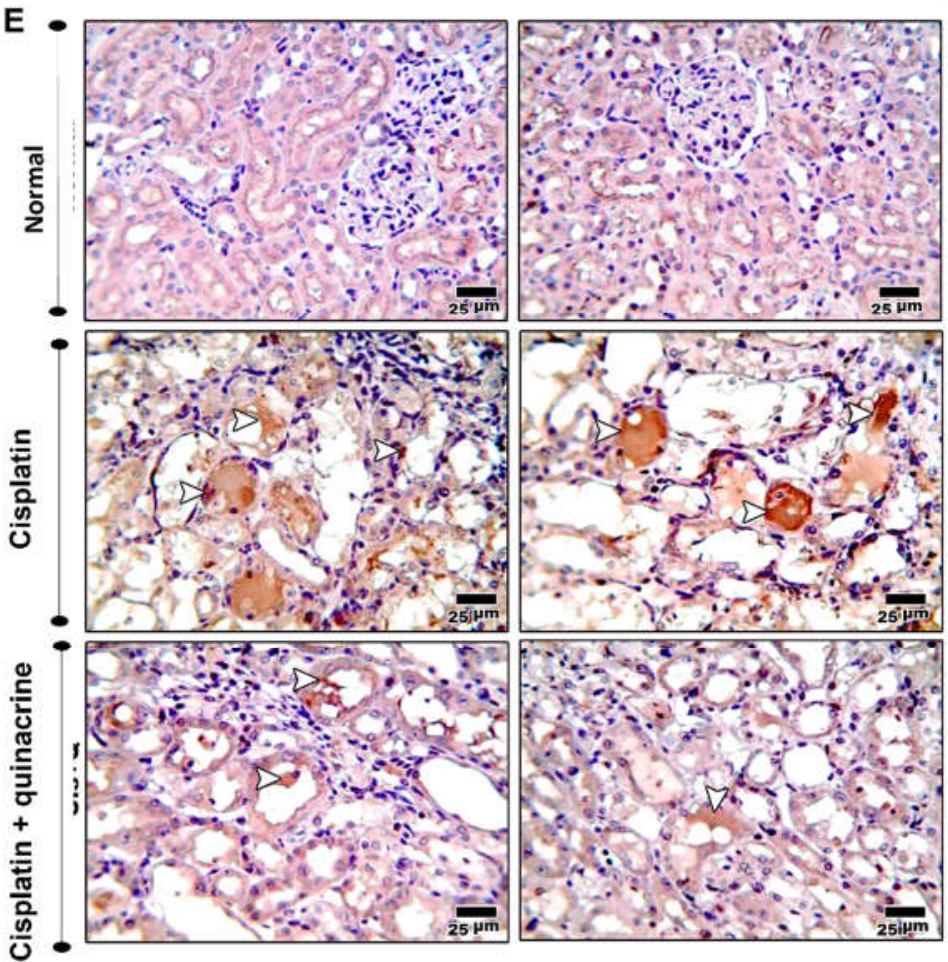

Figure 3. Quinacrine attenuated cisplatin-induced dysregulation of SIRT-1, ICAM-1 and TNF- $\alpha$. Effect of quinacrine administration (10 mg/kg/rat/ day: i.p) against cisplatin-induced nephrotoxicity in rats on (A) renal sirtuin-1 (SIRT-1) concentration $(n=7-8)$, (B) renal intercellular adhesion molecule-1 (ICAM-1) concentration $(n=7-8)$, (C) renal tumor necrosis factor- $\alpha$ (TNF- $\alpha)$ concentration $(n=7-8)$ and (D) \% area of TNF- $\alpha$ immunostaining $(n=6)$. (E) Microscopic pictures of immunostained renal sections against TNF- $\alpha$ showing negative expression in normal group and strong positive tubular expression appears in affected areas in renal sections from cisplatin group. The positive brown tubular expression decreased in renal sections from cisplatin + quinacrine group. (Arrowheads point to positive brown reaction.) High magnification $\mathrm{X}: 400$ bar $25 .{ }^{*}: p<0.05 ;{ }^{* *}: p<0.01$ and ${ }^{* * *} p<0.001$. Data are expressed as mean $\pm \mathrm{SEM}$.

\subsection{Quinacrine Attenuated Cisplatin-Induced Apoptosis}

Cisplatin caused apoptosis in renal tissue, which was indicated by the significant increase in immunostaining of apoptotic proteins BAX and p53 (Figure 4A,B), compared to the normal group. Additionally, immunostaining demonstrates that cisplatin significantly decreased the concentration of the anti-apoptotic protein BCL2 (Figure 4A,B), compared to the normal group. On the other hand, quinacrine significantly decreased the immunostaining area of apoptotic proteins BAX, p53 and significantly increased immunostaining area of anti-apoptotic protein BCL2 (Figure 4A,B), compared to the cisplatin group. These results are in accordance with the effect of cisplatin and quinacrine on caspases 1,3,8 and 9 (Supplementary Figure S1). 


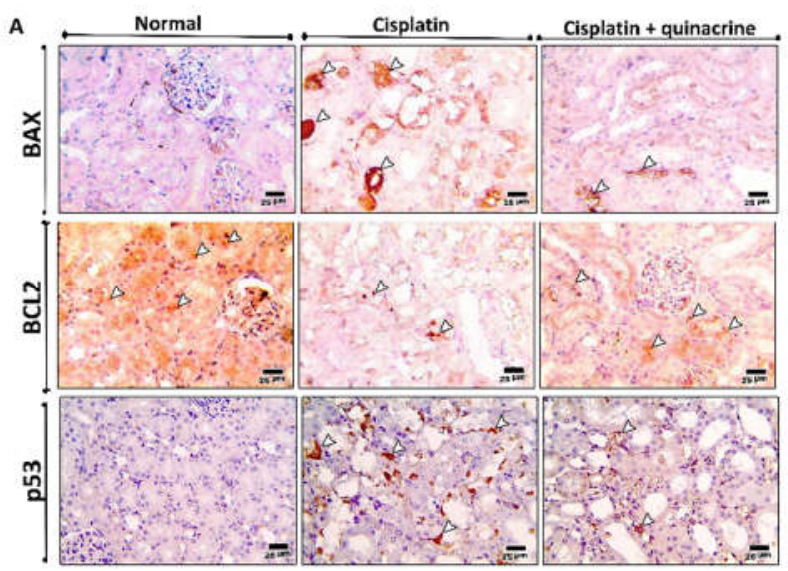

c
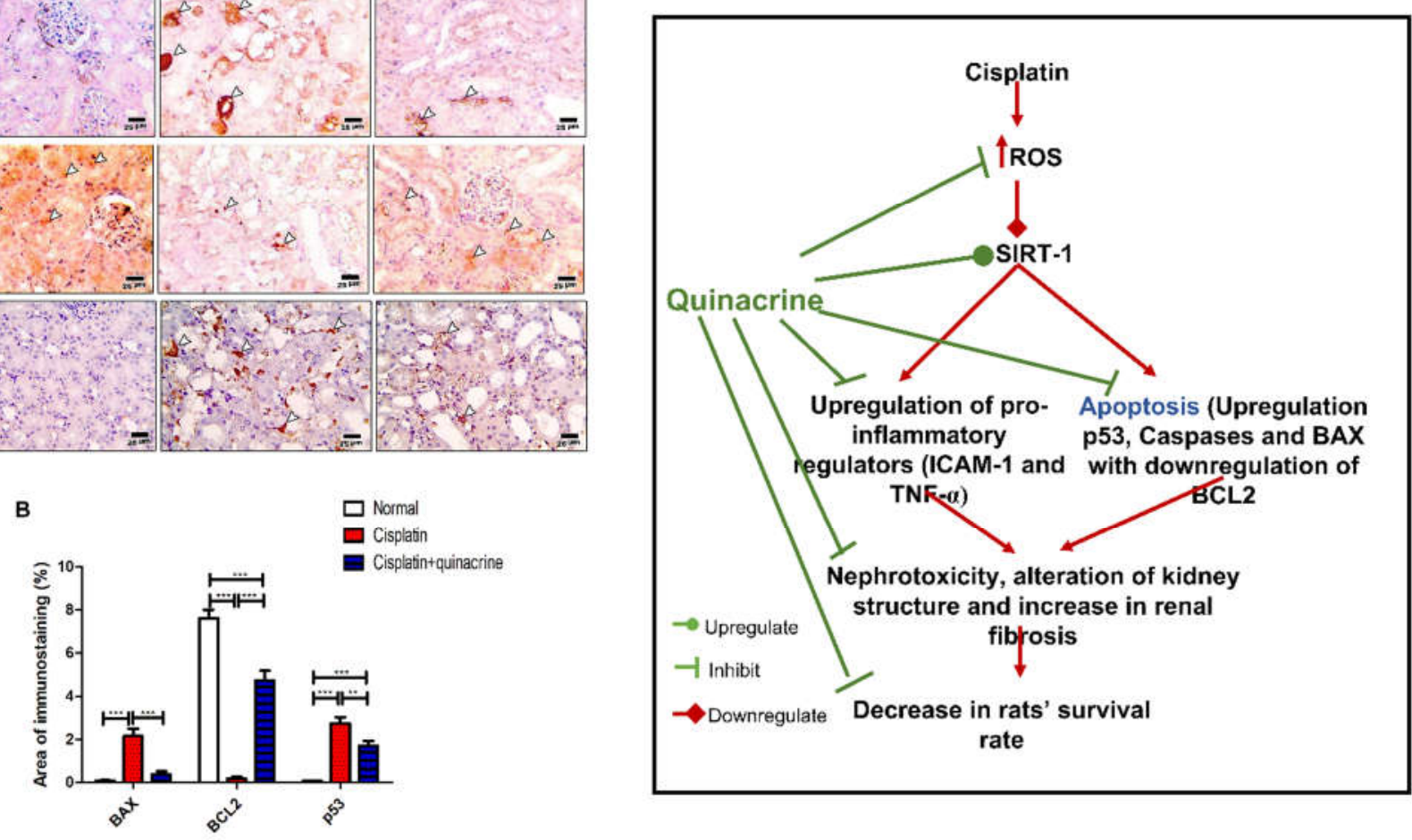

Figure 4. Quinacrine attenuated cisplatin-induced increase in apoptotic proteins and decrease in antiapoptotic protein. (A) Microscopic pictures of immunostained renal sections against BAX, BCL2 and p53; arrowheads point to positive brown reaction; high magnification X:400 bar 25. (B) Area of immunostaining of BAX, BCL2 and p53 (\%) $(n=6) .{ }^{* *}: p<0.01$, ${ }^{* * *}: p<0.001$. (C) Schematic diagram showing potential pathway of quinacrine's renoprotective effect against cisplatininduced toxicity. Data are expressed as mean \pm SEM.

\section{Discussion}

Nephrotoxicity is a serious side effect of cisplatin that limits its clinical use. This study aims to investigate the potential protective effect of quinacrine, an FDA-approved drug, against cisplatin-induced nephrotoxicity, in addition to studying the possible underlying molecular mechanism of quinacrine's action.

Single injection of cisplatin in rats induced nephrotoxicity, which was evident by the increase in mortality rate, relative kidney to body weight ratio, serum creatinine and urea concentrations, fibrosis formation and structural alterations of renal cortex and medulla. This was in agreement with El-Sherbiny et al. [16]. Moreover, cisplatin treatment led to a significant increase in renal MDA concentration and a decrease in TAC, which indicates cisplatin-induced oxidative stress in renal tissue. These results were consistent with previous studies that reported similar outcomes of cisplatin toxicity [17-19]. Quinacrine efficiently reversed cisplatin-induced nephrotoxicity phenotype. Previous studies show similar protective effects of quinacrine against in vivo cyclosporine-induced [20] or glycerolinduced nephrotoxicity [21].

Quinacrine was also previously reported to have an antioxidant effect in ulcerative colitis [22], status epilepticus [23] and neurodegenerative diseases [24]. In context, our results showed that quinacrine plays an antioxidant role by blocking cisplatin-induced oxidative stress in kidney. This was demonstrated by a significant decrease in renal MDA concentration and an increase in renal TAC. Furthermore, we studied the role of quinacrine in modulating SIRT- 1 and its underlying inflammatory and apoptosis pathways. Our data has shown that cisplatin caused a significant downregulation of renal tissue levels of SIRT-1, which was reversed by quinacrine. These results confirm the regulatory role of SIRT- 1 in drug-induced renal injuries [25]. While the modulatory effect of cisplatin of SIRT-1 was 
previously reported [9-11,26,27], this work is the first, to the best of our knowledge, to report the effect of quinacrine on SIRT-1 regulation.

As previously shown, inflammation plays a major role in the pathogenesis of cisplatininduced renal injury [28,29]. Consistent with previous work [29-31], our results showed that cisplatin evoked a marked pro-inflammatory response, which was indicated by the significant increase in renal levels of inflammatory mediators ICAM- 1 and TNF- $\alpha$. Importantly, quinacrine successfully reduced cisplatin-upregulated levels of ICAM-1 and TNF- $\alpha$. These results were consistent with different studies that reported the inhibitory effect of quinacrine on ICAM-1 [32] and TNF- $\alpha$ expression [33].

Recent studies revealed that SIRT-1 upregulation alleviates apoptosis in drug-induced injuries [34-36]. Apoptosis causes DNA fragmentation, which leads to increased oxidative stress and subsequent inflammation [34,37]. p53, a sensor of DNA damage and cell death, promotes apoptosis by regulating expression of caspases enzymes and BCL2 family members, all of which were reported to highly modulate cisplatin-induced apoptosis $[28,38]$. Among caspases, caspase-8 is an important mediator for an inflammatory cell death process known as necroptosis $[39,40]$.

In this study, cisplatin led to a significant increase in expression levels of caspases, BAX and p53 with a significant decrease in expression levels of BCL2. These results suggest that SIRT-1 downregulation might play a regulatory role in cisplatin-induced nephrotoxicity. Consistently, Kim et al. (2019) stated that SIRT-1 activation attenuates cisplatin-induced cell apoptosis, probably through deacetylating p53 [11]. Quinacrine blocked cisplatin-induced apoptosis by upregulating SIRT-1. Quinacrine has also been reported to have anti-apoptotic effects both in vitro [41] and in vivo [42]. Indeed, our data suggest that quinacrine inhibits cisplatin-induced apoptosis and necroptosis processes. Further investigation is needed to establish whether the inhibitory effect of quinacrine on both apoptosis and necroptosis is synergistic. The impact of such synergy was shown to be effective in alleviating cisplatin-induced nephrotoxicity [43].

Altogether, cisplatin downregulated SIRT-1 levels, which might be-in part-due to induction of oxidative stress. SIRT- 1 downregulation resulted in an increase in proinflammatory markers (ICAM- 1 and TNF- $\alpha$ ) in addition to upregulation of p53 and pro-apoptosis proteins (BAX and caspases). It also led to downregulation of the antiapoptosis protein BCL2. Quinacrine reversed these effects, which efficiently protected against cisplatin-induced nephrotoxicity (Figure 4C).

\section{Materials and Methods}

\subsection{Animals}

Twenty-nine male Sprague Dawley rats weighing (180-200 gm) were purchased from the Faculty of Pharmacy, Mansoura University, Egypt. Rats were allowed to be acclimated by keeping them under standard conditions of a $12 \mathrm{~h}$ dark and $12 \mathrm{hr}$ light cycle, $23-27^{\circ} \mathrm{C}$ temperature, and $48-52 \%$ humidity for a week before administration of the first dose. Rats were provided with the standard pellet diet and water ad libitum. The animal care and experiments described in this study complied with "Research Ethics Committee" Faculty of Medicine, Mansoura University, Mansoura, Egypt, which is in accordance with "Principles of Laboratory Animal Care" (NIH publication No. 85-23, revised 1985) in animal care and experiments.

\subsection{Study Medications}

Cisplatin (232120) and quinacrine (Q3251) were purchased from Sigma-Aldrich (Saint Louis, MO, USA). All other chemicals used in the study were of high purity and analytical grade.

\subsection{Induction of Cisplatin Toxicity}

Cisplatin was dissolved in saline. Acute renal toxicity was induced by intraperitoneal injection of $10 \mathrm{mg} / \mathrm{kg}$ cisplatin, a single dose, at day 5 [16,44]. 


\subsection{Experimental Model}

Rats were randomly divided into three groups:

1. Normal group (8 rats): rats were injected with $0.2 \mathrm{~mL}$ saline intraperitoneally, daily for 10 days.

2. Cisplatin group (13 rats): rats were intraperitoneally injected with $0.2 \mathrm{~mL}$ saline daily for 10 days, except at day 5 , when they were injected with cisplatin single-dose intraperitoneally $(10 \mathrm{mg} / \mathrm{kg})$.

3. Cisplatin + quinacrine ( 8 rats): rats were intraperitoneally injected with quinacrine (10 mg/kg/day) for 10 days [20,21,42,45] and were injected with a cisplatin single dose $(10 \mathrm{mg} / \mathrm{kg})$ at day 5 an hour after quinacrine treatment.

The mortality of the rats was daily recorded (Supplementary Table S1). At the end of the model, twenty-four hours after the last quinacrine injection, the rats were weighed. Blood samples $(5 \mathrm{~mL})$ were withdrawn via retro-orbital puncture under light ether anesthesia. Blood samples were allowed to coagulate, centrifuged, and then serum was aliquoted for further biochemical analyses.

Rats were sacrificed to dissect the kidneys. The kidneys were rinsed with cold phosphate buffer saline (PBS) pH:7.4, blotted dry with filter paper, and then weighed. A transverse cut of the right kidney was fixed in 10\% phosphate-buffered formalin (PBF) for histopathological and immunohistochemical analyses. The left kidney was homogenized in cold PBS, centrifuged, and then the supernatant was aliquoted and kept at $-80^{\circ} \mathrm{C}$ for further oxidative stress and ELISA analyses.

\subsection{Serum Biochemical Analysis}

Serum was used for the determination of creatinine (\#10053) and urea (\#10505) concentrations, according to Bartles et al., [46] and Fawcett el al., [47], respectively, using commercially available colorimetric kits (Human Co, Wiesbaden, Germany).

\subsection{Renal Oxidative Stress Analysis}

A supernatant of the kidney tissue homogenates was used for determination of the MDA (\# MD 25 29) concentration, following the method of Kei et al. [48] and TAC (\# TA 25 13), following the method of Koracevic et al. [49], using commercially available colorimetric kits (Biodiagnostic company, Cairo, Egypt).

\subsection{Renal ELISA Analysis}

A supernatant of the kidney tissue homogenates was used for determination of SIRT-1, ICAM- 1 , and TNF- $\alpha$ concentrations, according to the manufacturer's instructions, using the following ELISA kits: CSB-EL021339RA, CSB-E04576r and CSB-E11987r, respectively (Cusabio, Wuhan, China). Briefly, standards and samples were added to a 96-well plate, covered with adhesive strip, and incubated for $2 \mathrm{~h}$ at 37 degrees. Liquids were removed from all wells without washing. Biotin-antibody $(1 \times)$ was added to each well, covered with a new adhesive strip and incubated for $1 \mathrm{~h}$ at $37^{\circ} \mathrm{C}$. The antibody was removed and the wells were washed three times, using the provided washing buffer. HRP-avidin $(1 \times)$ was added to each well, and the microtiter plate was covered with a new adhesive strip and incubated for $1 \mathrm{~h}$ at $37^{\circ} \mathrm{C}$. The solution was aspirated, and the wells were washed five times. TMB substrate was added to each well and incubated for $20 \mathrm{~min}$ at $37^{\circ} \mathrm{C}$ in dark. STOP Solution was added to each well, and the optical density was measured, using a microplate reader at $450 \mathrm{~nm}$.

\subsection{Histopathological and Immunohistochemical Analyses}

The formalin-fixed kidney tissues were embedded in paraffin. Sections were cut into $5 \mu \mathrm{m}$ in thickness and stained with hematoxylin and eosin (H\&E). Slides were used for renal morphological and structural alteration examination under light microscopy. Pictures were captured, using a digital camera. The slides were blindly examined. 
For \% area of fibrosis, the sections were stained with Masson's trichrome for examining the fibrosis formation and assessment of \% area of fibrosis, as Masson's trichrome stains fibrotic areas with blue and parenchymal cells with red.

For immunohistochemical analysis, the sections were deparaffinized, rehydrated, and immersed in antigen retrieval solution (EDTA solution, $\mathrm{pH} 8$ ). The sections were treated with $3 \%$ hydrogen peroxide and protein block, followed by overnight incubation at $4{ }^{\circ} \mathrm{C}$ with the following antibodies: TNF- $\alpha$ (A0277: ABclonal, Woburn, MA, USA), Caspase-1 (sc-392736: Santa Cruz Biotechnology, INC., Heidelberg, Germany), Caspase-3 (GB11532: servicebio, Wuhan, China), Caspase-8 (CBS-PA001234: Cusabio, Wuhan, China), Caspase9 (CSB-PA001235: Cusabio, Wuhan, China), BAX (GB11007-1: Servicebio, Wuhan, China), BCL2 (61-0005-2: Genemed, San Francisco, CA, USA) and p53 (A11232: ABclonal, Woburn, MA, USA) at a 1:100 dilution factor. The slides were rinsed three times with PBS, and incubated with appropriate secondary antibodies for $30 \mathrm{~min}$ at $25^{\circ} \mathrm{C}$. As a negative control, the primary antibody was replaced by normal rat serum. The specificity of the used antibodies was checked using No antibody control, secondary anti-mouse and secondary anti-rabbit controls are showing negative staining to confirm that the reported signal was antigen specific (Supplementary Figure S2). For the analysis of antibody binding, a diaminobenzidine kit was used against the H\&E counterstain. Finally, the slides were checked using light microscopy to detect the distinct brown-colored reaction.

The slides for Masson's trichrome and immune-histochemical stainings were photographed, using an Olympus ${ }^{\circledR}$ digital camera installed on an Olympus ${ }^{\circledR}$ microscope with $1 / 2 \times$ photo adaptor, using $400 \times$ objective. The images were then analyzed on an Intel ${ }^{\circledR}$ Core I $3^{\circledR}$ based computer, using VideoTest Morphology ${ }^{\circledR}$ software (St.-Petersburg, Russia) with a specific built-in routine for area, \% area measurement and object counting. Masson's trichrome blue color stained areas, Caspase- $1,-3,-8$, and -9 , and BCL2 and p53 brown color immunostained areas were blindly determined, using ImageJ software (National Institutes of Health, Bethesda, MD, USA), taking six reads for each section at $400 \times$ magnification. Briefly, digital image analysis was performed by uniformly adjusting size of all images to 12.7 centimeters in width and 9 centimeters in length ( $300 \mathrm{dpi})$. In this case, a scale bar was set to (25 microns). All images were loaded into the (image J) program, where the 6 fields were investigated. Finally, the average area and \% area for each group were calculated relative to the control normal group.

\subsection{Statistical Analysis}

All data are presented as mean \pm standard error of the mean (S.E.M). Statistical analysis was performed via GraphPad Prism V 5.02 (GraphPad Software Inc., San Diego, CA, USA). The distribution of data was analyzed to check the parametric or non-parametric tests, which will follow. One-way ANOVA followed by Bartlett's test (post-test) was used to determine the statistical significance between groups. $p \leq 0.05$ was considered statistically significant, with the following used symbols: ${ }^{*} p<0.05 ;{ }^{* *} p<0.01$; ${ }^{* * *} p<0.001$.

\section{Conclusions}

The current study proposes, for the first time, that quinacrine ameliorated cisplatininduced renal toxicity via upregulation of SIRT-1. Moreover, no previous studies, to our best knowledge, have reported the effect of quinacrine on SIRT-1 regulation. Furthermore, quinacrine significantly downregulated the pro-inflammatory proteins (ICAM-1 and TNF- $\alpha$ ), restored the cisplatin-disrupted oxidative balance, suppressed the apoptotic mediators (caspases, BAX, and p53), and upregulated the anti-apoptotic protein BCL2. Although clinical studies are needed to establish the renoprotective effect of quinacrine against cisplatin-induced renal toxicity, this study provides evidence for a potential novel therapeutic use of quinacrine as a protective agent against cisplatin-induced renal injury. 
Supplementary Materials: The following are available online at https:/ / www.mdpi.com/article/10 .3390 /ijms221910660/s1, Figure S1: Quinacrine attenuated cisplatin-induced apoptosis., Figure S2: The specificity of the used antibodies for immunohistochemistry. Table S1: The number of rats in each group at day 0 (the start) and day 10 (the end) of the experimental model.

Author Contributions: Conceptualization, N.F.A.E.-M., H.A.E., M.E.-S. and N.H.E.; methodology, N.F.A.E.-M., H.A.E., M.E.-S. and N.H.E.; Statistical analysis, N.F.A.E.-M.; Experiments, N.H.E., N.F.A.E.-M.; histopathological and immunohistochemical analyses, H.A.E., M.E.-S.; formal analysis, N.F.A.E.-M., M.E.-S. and N.H.E.; writing—original draft preparation, N.F.A.E.-M.; writing-review and editing, N.F.A.E.-M., H.A.E., M.E.-S. and N.H.E. All authors have read and agreed to the published version of the manuscript.

Funding: This research was funded by the Deanship of Scientific Research at Princess Nourah bint Abdulrahman University through the Fast-track Research Funding Program.

Institutional Review Board Statement: The study was conducted according to the guidelines of the with "Principles of Laboratory Animal Care" (NIH publication No. 85-23, revised 1985) in the animal care and experiments and approved by "Research Ethics Committee" Faculty of Medicine, Mansoura University, Mansoura, Egypt.

Informed Consent Statement: Not applicable.

Data Availability Statement: The data that support the findings of this study are available from the authors upon reasonable request.

Acknowledgments: The authors extend their appreciation to AlMaarefa University for supporting this research.

Conflicts of Interest: The authors declare no conflict of interest.

\section{References}

1. Cohen, S.M.; Lippard, S.J. Cisplatin: From DNA damage to cancer chemotherapy. Prog. Nucleic Acid Res. Mol. Biol. 2001, 67, 93-130. [PubMed]

2. Pabla, N.; Dong, Z. Cisplatin nephrotoxicity: Mechanisms and renoprotective strategies. Kidney Int. 2008, 73, 994-1007. [CrossRef] [PubMed]

3. Wang, D.; Lippard, S.J. Cellular processing of platinum anticancer drugs. Nat. Rev. Drug Discov. 2005, 4, 307-320. [CrossRef] [PubMed]

4. Yin, M.; Li, N.; Makinde, E.A.; Olatunji, O.J.; Ni, Z. N6-2-hydroxyethyl-adenosine ameliorate cisplatin induced acute kidney injury in mice. All Life 2020, 13, 244-251. [CrossRef]

5. Maimaitiyiming, H.; Li, Y.; Cui, W.; Tong, X.; Norman, H.; Qi, X.; Wang, S. Increasing cGMP-dependent protein kinase I activity attenuates cisplatin-induced kidney injury through protection of mitochondria function. Am. J. Physiol. Ren. Physiol. 2013, 305, F881-F890. [CrossRef]

6. Khan, M.A.; Liu, J.; Kumar, G.; Skapek, S.X.; Falck, J.R.; Imig, J.D. Novel orally active epoxyeicosatrienoic acid (EET) analogs attenuate cisplatin nephrotoxicity. FASEB J. 2013, 27, 2946-2956. [CrossRef]

7. Yu, X.; Meng, X.; Xu, M.; Zhang, X.; Zhang, Y.; Ding, G.; Huang, S.; Zhang, A.; Jia, Z. Celastrol ameliorates cisplatin nephrotoxicity by inhibiting NF- $\mathrm{BB}$ and improving mitochondrial function. EBioMedicine 2018, 36, 266-280. [CrossRef]

8. Chao, C.S.; Tsai, C.S.; Chang, Y.P.; Chen, J.M.; Chin, H.K.; Yang, S.C. Hyperin inhibits nuclear factor kappa B and activates nuclear factor E2-related factor-2 signaling pathways in cisplatin-induced acute kidney injury in mice. Int. Immunopharmacol. 2016, 40, 517-523. [CrossRef]

9. Deng, J.-S.; Jiang, W.-P.; Chen, C.-C.; Lee, L.-Y.; Li, P.-Y.; Huang, W.-C.; Liao, J.-C.; Chen, H.-Y.; Huang, S.-S.; Huang, G.-J. Cordyceps cicadae Mycelia Ameliorate Cisplatin-Induced Acute Kidney Injury by Suppressing the TLR4/NF- $\mathrm{kB} / \mathrm{MAPK}$ and Activating the HO-1/Nrf2 and Sirt-1/AMPK Pathways in Mice. Oxid. Med. Cell Longev. 2020, 2020, 7912763. [CrossRef]

10. Kim, D.H.; Jung, Y.J.; Lee, J.E.; Lee, A.S.; Kang, K.P.; Lee, S.; Park, S.K.; Han, M.K.; Lee, S.Y.; Ramkumar, K.M.; et al. SIRT1 activation by resveratrol ameliorates cisplatin-induced renal injury through deacetylation of p53. Am. J. Physiol.-Ren. Physiol. 2011, 301, F427-F435. [CrossRef]

11. Kim, J.-Y.; Jo, J.; Kim, K.; An, H.-J.; Gwon, M.-G.; Gu, H.; Kim, H.-J.; Yang, A.Y.; Kim, S.-W.; Jeon, E.J.; et al. Pharmacological Activation of Sirt1 Ameliorates Cisplatin-Induced Acute Kidney Injury by Suppressing Apoptosis, Oxidative Stress, and Inflammation in Mice. Antioxidants 2019, 8, 322. [CrossRef]

12. Qiao, J.; Shuai, Y.; Zeng, X.; Xu, D.; Rao, S.; Zeng, H.; Li, F. Comparison of Chemical Compositions, Bioactive Ingredients, and In Vitro Antitumor Activity of Four Products of Cordyceps (Ascomycetes) Strains from China. Int. J. Med. Mushrooms 2019, 21, 331-342. [CrossRef]

13. Oien, D.B.; Pathoulas, C.L.; Ray, U.; Thirusangu, P.; Kalogera, E.; Shridhar, V. Repurposing quinacrine for treatment-refractory cancer. Semin. Cancer Biol. 2021, 68, 21-30. [CrossRef] [PubMed] 
14. Yan, D.; Borucki, R.; Sontheimer, R.D.; Werth, V.P. Candidate drug replacements for quinacrine in cutaneous lupus erythematosus. Lupus Sci. Med. 2020, 7, e000430. [CrossRef]

15. Ochsendorf, F.R. Use of antimalarials in dermatology. J. Dtsch. Dermatol. Ges. 2010, 8, 829-844; quiz 845. [CrossRef] [PubMed]

16. Elsherbiny, N.M.; Eladl, M.A.; Al-Gayyar, M.M.H. Renal protective effects of arjunolic acid in a cisplatin-induced nephrotoxicity model. Cytokine 2016, 77, 26-34. [CrossRef] [PubMed]

17. Badawy, A.M.; El-Naga, R.N.; Gad, A.M.; Tadros, M.G.; Fawzy, H.M. Wogonin pre-treatment attenuates cisplatin-induced nephrotoxicity in rats: Impact on PPAR- $\gamma$, inflammation, apoptosis and Wnt/ $\beta$-catenin pathway. Chem.-Biol. Interact. 2019, 308, 137-146. [CrossRef] [PubMed]

18. Gao, H.; Zhang, S.; Hu, T.; Qu, X.; Zhai, J.; Zhang, Y.; Tao, L.; Yin, J.; Song, Y. Omeprazole protects against cisplatin-induced nephrotoxicity by alleviating oxidative stress, inflammation, and transporter-mediated cisplatin accumulation in rats and HK-2 cells. Chem.-Biol. Interact. 2019, 297, 130-140. [CrossRef] [PubMed]

19. Wang, S.W.; Xu, Y.; Weng, Y.Y.; Fan, X.Y.; Bai, Y.F.; Zheng, X.Y.; Lou, L.J.; Zhang, F. Astilbin ameliorates cisplatin-induced nephrotoxicity through reducing oxidative stress and inflammation. Food Chem. Toxicol. 2018, 114, 227-236. [CrossRef] [PubMed]

20. Khader, A.A.; Sulaiman, M.A.; Kishore, P.N.; Morais, C.; Tariq, M. Quinacrine Attenuates Cyclosporine-Induced Nephrotoxicity in Rats1. Transplantation 1996, 62, 427-435. [CrossRef] [PubMed]

21. Al Asmari, A.K.; Al Sadoon, K.T.; Obaid, A.A.; Yesunayagam, D.; Tariq, M. Protective effect of quinacrine against glycerol-induced acute kidney injury in rats. BMC Nephrol. 2017, 18, 41. [CrossRef] [PubMed]

22. Chumanevich, A.A.; Witalison, E.E.; Chaparala, A.; Chumanevich, A.; Nagarkatti, P.; Nagarkatti, M.; Hofseth, L.J. Repurposing the anti-malarial drug, quinacrine: New anti-colitis properties. Oncotarget 2016, 7, 52928-52939. [CrossRef]

23. Ahmad, M.; Abu-Taweel, G.M.; Aboshaiqah, A.E.; Ajarem, J.S. The effects of quinacrine, proglumide, and pentoxifylline on seizure activity, cognitive deficit, and oxidative stress in rat lithium-pilocarpine model of status epilepticus. Oxid. Med. Cell Longev. 2014, 2014, 630509. [CrossRef] [PubMed]

24. Turnbull, S.; Tabner, B.J.; Brown, D.R.; Allsop, D. Quinacrine acts as an antioxidant and reduces the toxicity of the prion peptide PrP106-126. Neuroreport 2003, 14, 1743-1745. [CrossRef] [PubMed]

25. Chong, Z.Z.; Shang, Y.C.; Wang, S.; Maiese, K. SIRT1: New avenues of discovery for disorders of oxidative stress. Expert Opin. Ther. Targets 2012, 16, 167-178. [CrossRef] [PubMed]

26. Qi, Z.; Li, Z.; Li, W.; Liu, Y.; Wang, C.; Lin, H.; Liu, J.; Li, P. Pseudoginsengenin DQ Exhibits Therapeutic Effects in CisplatinInduced Acute Kidney Injury via Sirt1/NF-kB and Caspase Signaling Pathway without Compromising Its Antitumor Activity in Mice. Molecules 2018, 23, 3038. [CrossRef] [PubMed]

27. Ali, F.E.M.; Hassanein, E.H.M.; El-Bahrawy, A.H.; Omar, Z.M.M.; Rashwan, E.K.; Abdel-Wahab, B.A.; Abd-Elhamid, T.H. Nephroprotective effect of umbelliferone against cisplatin-induced kidney damage is mediated by regulation of NRF2, cytoglobin, SIRT1/FOXO-3, and NF- kB-p65 signaling pathways. J. Biochem. Mol. Toxicol. 2021, 35, e22738. [CrossRef]

28. Miller, R.P.; Tadagavadi, R.K.; Ramesh, G.; Reeves, W.B. Mechanisms of Cisplatin nephrotoxicity. Toxins 2010, 2, 2490-2518. [CrossRef]

29. El-Naga, R.N. Pre-treatment with cardamonin protects against cisplatin-induced nephrotoxicity in rats: Impact on NOX-1, inflammation and apoptosis. Toxicol. Appl. Pharmacol. 2014, 274, 87-95. [CrossRef]

30. Ramesh, G.; Reeves, W.B. TNF-alpha mediates chemokine and cytokine expression and renal injury in cisplatin nephrotoxicity. J. Clin. Investig. 2002, 110, 835-842. [CrossRef]

31. Ueki, M.; Ueno, M.; Morishita, J.; Maekawa, N. D-ribose ameliorates cisplatin-induced nephrotoxicity by inhibiting renal inflammation in mice. Tohoku J. Exp. Med. 2013, 229, 195-201. [CrossRef]

32. Harada, M.; Morimoto, K.; Kondo, T.; Hiramatsu, R.; Okina, Y.; Muko, R.; Matsuda, I.; Kataoka, T. Quinacrine Inhibits ICAM-1 Transcription by Blocking DNA Binding of the NF- $\kappa B$ Subunit p65 and Sensitizes Human Lung Adenocarcinoma A549 Cells to TNF- $\alpha$ and the Fas Ligand. Int. J. Mol. Sci. 2017, 18, 2603. [CrossRef]

33. Alves, P.; Bashir, M.M.; Wysocka, M.; Zeidi, M.; Feng, R.; Werth, V.P. Quinacrine Suppresses Tumor Necrosis Factor- $\alpha$ and IFN- $\alpha$ in Dermatomyositis and Cutaneous Lupus Erythematosus. J. Investig. Dermatol. Symp. Proc. 2017, 18, S57-S63. [CrossRef]

34. Yan, T.; Huang, J.; Nisar, M.F.; Wan, C.; Huang, W. The Beneficial Roles of SIRT1 in Drug-Induced Liver Injury. Oxid. Med. Cell Longev. 2019, 2019, 8506195. [CrossRef]

35. El Kiki, S.M.; Omran, M.M.; Mansour, H.H.; Hasan, H.F. Metformin and/or low dose radiation reduces cardiotoxicity and apoptosis induced by cyclophosphamide through SIRT-1/SOD and BAX/Bcl-2 pathways in rats. Mol. Biol. Rep. 2020, 47, 5115-5126. [CrossRef]

36. Wang, T.; Li, X.; Sun, S.L. EX527, a Sirt-1 inhibitor, induces apoptosis in glioma via activating the p53 signaling pathway. Anti-Cancer Drugs 2020, 31, 19-26. [CrossRef]

37. Ismail, A.F.; Salem, A.A.; Eassawy, M.M. Hepatoprotective effect of grape seed oil against carbon tetrachloride induced oxidative stress in liver of $\gamma$-irradiated rat. J. Photochem. Photobiol. B Biol. 2016, 160, 1-10. [CrossRef] [PubMed]

38. Bassett, E.A.; Wang, W.; Rastinejad, F.; El-Deiry, W.S. Structural and Functional Basis for Therapeutic Modulation of p53 Signaling. Clin. Cancer Res. 2008, 14, 6376-6386. [CrossRef] [PubMed]

39. Oberst, A.; Dillon, C.P.; Weinlich, R.; McCormick, L.L.; Fitzgerald, P.; Pop, C.; Hakem, R.; Salvesen, G.S.; Green, D.R. Catalytic activity of the caspase-8-FLIP L complex inhibits RIPK3-dependent necrosis. Nature 2011, 471, 363-367. [CrossRef] [PubMed]

40. Ch'en, I.L.; Tsau, J.S.; Molkentin, J.D.; Komatsu, M.; Hedrick, S.M. Mechanisms of necroptosis in T cells. J. Exp. Med. 2011, 208, 633-641. [CrossRef] 
41. Shin, S.; Kim, Y.B.; Hur, G.H. Involvement of phospholipase A2 activation in anthrax lethal toxin-induced cytotoxicity. Cell Biol. Toxicol. 1999, 15, 19-29. [CrossRef] [PubMed]

42. Kencebay, C.; Derin, N.; Ozsoy, O.; Kipmen-Korgun, D.; Tanriover, G.; Ozturk, N.; Basaranlar, G.; Yargicoglu-Akkiraz, P.; Sozen, B.; Agar, A. Merit of quinacrine in the decrease of ingested sulfite-induced toxic action in rat brain. Food Chem. Toxicol. 2013, 52, 129-136. [CrossRef] [PubMed]

43. Tristão, V.R.; Pessoa, E.A.; Nakamichi, R.; Reis, L.A.; Batista, M.C.; de Souza Durão Junior, M.; Monte, J.C.M. Synergistic effect of apoptosis and necroptosis inhibitors in cisplatin-induced nephrotoxicity. Apoptosis 2016, 21, 51-59. [CrossRef]

44. Adaramoye, O.A.; Azeez, A.F.; Ola-Davies, O.E. Ameliorative Effects of Chloroform Fraction of Cocos nucifera L. Husk Fiber Against Cisplatin-induced Toxicity in Rats. Pharmacogn. Res. 2016, 8, 89-96. [CrossRef]

45. Hirose, Y.; Tabuchi, K.; Oikawa, K.; Murashita, H.; Sakai, S.; Hara, A. The effects of the glucocorticoid receptor antagonist RU486 and phospholipase A2 inhibitor quinacrine on acoustic injury of the mouse cochlea. Neurosci. Lett. 2007, 413, 63-67. [CrossRef]

46. Bartels, H.; Böhmer, M.; Heierli, C. Serum creatinine determination without protein precipitation. Clin. Chim. Acta Int. J. Clin. Chem. 1972, 37, 193-197. [CrossRef]

47. Fawcett, J.K.; Scott, J.E. A rapid and precise method for the determination of urea. J. Clin. Pathol. 1960, 13, 156-159. [CrossRef] [PubMed]

48. Kei, S. Serum lipid peroxide in cerebrovascular disorders determined by a new colorimetric method. Clin. Chim. Acta 1978, 90, 37-43. [CrossRef]

49. Koracevic, D.; Koracevic, G.; Djordjevic, V.; Andrejevic, S.; Cosic, V. Method for the measurement of antioxidant activity in human fluids. J. Clin. Pathol. 2001, 54, 356. [CrossRef] 